\title{
Xenotransplantation of pig islets into Mexican children: Were the fundamental ethical requirements to proceed with such a study really met?
}

\author{
Megan Sykes and Emanuele Cozzi ${ }^{1}$ \\ Transplantation Biology Research Center, Massachusetts General Hospital/Harvard Medical School, MGH-East, 13th Street, Boston, MA O2129, USA \\ and ${ }^{1}$ Ospedale Giustinianeo, Via Giustiniani 2, Padova, Italy \\ (Correspondence should be addressed to E Cozzi; Email: emanuele.cozzi@unipd.it)
}

European Journal of Endocrinology 154 921-922

We are writing on behalf of the Council of the International Xenotransplantation Association (IXA) and its Ethics Committee, following the recent publication in the European Journal of Endocrinology by Valdés-González and colleagues of the data relating to a xenotransplantation trial in children (1).

The IXA is the major international professional/ scientific organization in the field of xenotransplantation. We are a section of the Transplantation Society (TTS). In addition to our scientific mission, one of the IXA's major goals is to promote the responsible and ethical conduct of clinical and animal research in our field. In so doing, we hope to ensure that possible clinical studies in xenotransplantation are fully justified and do not elicit criticisms that may ultimately jeopardize the tremendous efforts made by many individuals to move this promising field forward.

In this context, we draw your attention to some serious concerns regarding the study in Valdés-González et al.'s article cited above. We are concerned that this study may not have been conducted in keeping with the ethical principles that the IXA has adopted for clinical studies in xenotransplantation, as published in an earlier position paper by our Ethics Committee (2) and reprinted in the TTS official journal, Transplantation (3). These principles are aimed in part at minimizing the potential risk to society of new infections that could be transmitted, particularly if xenotransplantation is conducted using source animals that are not adequately quality-controlled and if xenotransplant recipients are not properly monitored.

In particular, we are concerned that this study was undertaken in the complete absence of convincing efficacy data of the approach in preclinical large animal models. As xenotransplantation procedures may expose patients, and possibly society at large, to unknown potential infectious risks, one of our guiding principles is that there should be a high expectation of benefit, based on preclinical data, before such risks can be considered acceptable in clinical trials. Based on our knowledge of the literature in this field, we feel that the necessary preclinical groundwork was not done prior to initiation of the above clinical study. The risk/benefit ratio comes further into question when one considers the availability of insulin therapy and allogeneic pancreas transplantation. In this situation, the xenotransplantation approach used cannot be viewed as a life-saving procedure.

In addition, it is unclear to us why this trial was conducted entirely in children, as type I diabetes is equally prevalent in adults. Because of the potential risks to society and resultant necessary constraints on xenotransplant recipients' behaviour, there are major ethical issues involved concerning the acquisition of informed consent from such patients. Due to biosafety reasons, national and international regulatory agencies who have considered the issue in depth have recommended that xenotransplantation trials should entail long-term patient monitoring and informing sexual contacts of the xenotransplant. In light of these severe limitations to an individual's freedom that should result from inclusion in such a study, we feel strongly that such trials should not currently involve minors, who cannot provide adequate informed consent. The complexities of obtaining informed consent requiring long-term commitments from patients, in apparent violation of the right of a study participant to withdraw at any time, has been the topic of intense discussion and consideration by the US Secretary's Advisory Committee on Xenotransplantation, whose report has recently been published (5). It is unclear how the scientific and safety uncertainties, ethical issues and necessary behavioral constraints associated with this approach could have been adequately conveyed to the parents who signed the informed consent in this trial. 
One of the principles adopted by the IXA is that clinical xenotransplantation should not be carried out without oversight from a national regulatory agency. The World Health Assembly (WHA) has recently adopted a resolution urging that this policy be followed worldwide (4). We are concerned that Mexico - the country where the study in question (1) was conducted -has not, to our knowledge, established regulatory procedures for overseeing and monitoring xenotransplantation that maintain the standards cited in the IXA Ethics Committee paper (2). We wonder whether your journal requested and obtained any assurance that appropriate monitoring and oversight was available for Valdes-Gonzalez et al.'s study.

We would also like to bring to your attention the outcome of recent discussions of the Council of the IXA regarding the publication in our own official journal, Xenotransplantation, of clinical studies from countries that may not have established national regulatory agencies with suitable guidelines to oversee xenotransplantation trials. A short statement summarizing our opinion appeared in the January 2006 issue of Xenotransplantation (6). The statement reiterates that of the Ethics Committee of the IXA as published in Xenotransplantation in 2003 (2). It reminds the scientific community that oversight from a national regulatory agency, with guidelines and monitoring procedures that meet the standards of those published by regulatory agencies such as those cited in the above Position Paper (2), are mandatory. Furthermore, local institutional review board approval and adequate informed consent are essential. Any submitted manuscript should clearly state whether the trial was carried out according to these standards, and whether it was overseen by an independent national authority. Our statement also indicates that, in countries where an appropriate national regulatory body is not available, it is incumbent upon the investigators to demonstrate awareness of the potential infectious risks of their trial and to show that they have taken appropriate measures to minimize such risks. It is also the authors' responsibility to indicate that they have followed the principles adopted by the IXA (2). For such studies, the supporting information will be carefully evaluated by the Ethics Committee of the IXA. In all cases, however, convincing efficacy data in preclinical primate studies is essential before proceeding with clinical trials.

We believe that the ethical principles outlined in the IXA statements should be adopted by all other scientific journals and we encourage the European Journal of Endocrinology to join us in implementing this policy for any article dealing with clinical xenotransplantation that may be submitted to the Journal in the future.

We are firmly convinced that only timely, well planned, scientifically rigorous and ethically acceptable clinical studies will provide the confidence within our society at large that will be required for this emerging therapy to meet its clinical potential.

\section{References}

1 Valdés-González RA, Dorantes LM, Garibay GN, Bracho-Blanchet E, Mendez AJ, Dávila-Pérez R, Elliott RB, Terán L \& White DJG. Xenotransplantation of porcine neonatal islets of Langerhans and Sertoli cells: a 4-year study. European Journal of Endocrinology 2005153 419-427.

2 Sykes M, d'Apice A \& Sandrin M. The ethics of xenotransplantation. Position paper of the Ethics Committee of the International Xenotransplantation Association. Xenotransplantation 200310 194-203.

3 Sykes M, d'Apice A \& Sandrin M. IXA Ethics Committee. Position paper of the Ethics Committee of the International Xenotransplantation Association. Transplantation 200478 1101-1107.

4 World Health Organization (http://www.who.int/transplantation/ xeno). Reported in Transplantation 200579 636-637.

5 U.S. Department Of Health And Human Services Secretary's Advisory Committee On Xenotransplantation. Informed Consent In Clinical Research Involving Xenotransplantation. Available at: http:// www4.od.nih.gov/oba/SACX/reports/IC_draft_030905.pdf.

6 Sykes M \& Cooper DKC. Letter to authors. Xenotransplantation 2006 132.

Received 9 February 2006

Accepted 14 February 2006 